\title{
On generalizations of the Petersen graph and the Coxeter graph
}

\author{
Marko Orel \\ University of Primorska, FAMNIT, Glagoljaška 8, 6000 Koper, Slovenia \\ IMFM, Jadranska 19, 1000 Ljubljana, Slovenia \\ University of Primorska, IAM, Muzejski trg 2, 6000 Koper, Slovenia \\ marko.orel@upr.si
}

Submitted: Sep 27, 2013; Accepted: Nov 4, 2015; Published: Nov 13, 2015

Mathematics Subject Classifications 2010: 05C50, 15B33, 15B57

\begin{abstract}
In this note we consider two related infinite families of graphs, which generalize the Petersen and the Coxeter graph. The main result proves that these graphs are cores. It is determined which of these graphs are vertex/edge/arc-transitive or distance-regular. Girths and odd girths are computed. A problem on hamiltonicity is posed.
\end{abstract}

Keywords: core; Petersen graph; Coxeter graph; hermitian matrix; symmetric matrix

\section{Introduction}

All graphs considered in this paper are finite undirected with no loops and multiple edges. A graph homomorphism between two graphs $\Gamma$ and $\Gamma^{\prime}$ is a map $\Phi: V(\Gamma) \rightarrow V\left(\Gamma^{\prime}\right)$ between the vertex sets such that $\{\Phi(u), \Phi(v)\}$ is an edge in $\Gamma^{\prime}$ whenever $\{u, v\}$ is an edge in $\Gamma$. In particular, $\Phi(u) \neq \Phi(v)$ for any edge $\{u, v\}$. A bijective graph homomorphism such that $\{\Phi(u), \Phi(v)\}$ is an edge if and only if $\{u, v\}$ is an edge is a graph isomorphism. When $\Gamma=\Gamma^{\prime}$, a graph homomorphism and a graph isomorphism are called a graph endomorphism and a graph automorphism, respectively. Recall that a bijective graph endomorphism is the same as a graph automorphism, since the graphs considered are finite. A graph is a core if all of its endomorphisms are automorphisms. A subgraph $\Gamma^{\prime}$ in a graph $\Gamma$ is a core of $\Gamma$, if it is a core and there exists some graph homomorphism $\Phi: \Gamma \rightarrow \Gamma^{\prime}$. Though every graph has a core, which is an induced graph and unique up to isomorphism [7, Lemma 6.2.2], it is not trivial to provide examples of cores. For some known classes and for the general introduction to this subject we refer to [7]. Provided 
that the automorphism group of a graph acts transitively on non-edges, it was recently shown that the graph is either a core or it has a complete core [4, Corollary 2.2]. Same type of a result was obtained for connected regular graphs, where the automorphism group acts transitively on pairs of vertices at distance two [8, Theorem 4.1]. This property is shared by distance-transitive graphs. In both papers [4] and [8] it is emphasized that it is often difficult to decide which of the two possibilities about the core occurs. For the point graph of a classical polar space or for its complement, the problem actually translates on deciding whether the polar space has an ovoid, spread, and a partition into ovoids [4, Theorem 3.5]. This is a well known problem in finite geometry, which is still unsolved in general. Nevertheless, for several classes of graphs in $[4,8]$ the problem of whether the graph is a core or it has a complete core is solved. In particular, distance-regular non-bipartite graphs with no triangles are cores [8, Corollary 4.8].

Several matrix preserver problems demand a characterization of maps, defined on a particular set of matrices, which preserve a given binary relation. If the considered matrices have coefficients in a finite field, the preservers can be interpreted as endomorphisms of a finite graph. If it turns out that the graph is a core, its endomorphisms are bijective and their characterization is easier to obtain. The maps $\Phi$, defined on the set of all hermitian matrices over a finite field, for which the rank rk $(\Phi(A)-\Phi(B))$ equals one whenever $\operatorname{rk}(A-B)$ is one, were characterized in [12], by showing that the corresponding graph is a core. The proof was later shortened in the thesis [16] by applying [8, Theorem 4.1]. Analogous result for symmetric matrices over a finite field was obtained in [15]. Here the graph is a core, unless we consider $2 \times 2$ symmetric matrices in which case the core of a graph is complete. Very recently, the present author showed that the graph, which is formed by invertible hermitian matrices over a field $\mathbb{F}_{q^{2}}$ with $q^{2}$ elements, is a core, provided that $q \geqslant 4$ [13]. In this paper we prove the same result for $q=2$. The proof demands different techniques from those in [13], since the graph is without triangles when $q=2$. In contrast, when $q \geqslant 3$, any two adjacent vertices determine a maximal clique on either $q$ or $q-1$ vertices. The techniques developed in this paper allows us to prove also that the graph formed by invertible binary symmetric matrices is a core. An extra motivation to study the two families of graphs - formed by invertible hermitian matrices over $\mathbb{F}_{4}$ or by invertible symmetric matrices over $\mathbb{F}_{2}$ - is the fact that they generalize the well known Petersen and Coxeter graph, respectively. These two graphs are already known to be cores [7, p. 125].

The paper is organized as follows. In Section 2 we provide the notation and list few auxiliary theorems that are used in the proofs. The statement and the proofs of the main results are given in Section 3. In Section 4 we determine which of the considered graphs are vertex-transitive, edge-transitive, arc-transitive, or distance-regular, and state a problem on hamiltonicity that is related to a variant of the Lovász problem (cf. [9]).

\section{Preliminaries}

Let $\mathbb{F}_{4}=\{0,1, \imath, 1+\imath\}$ be the field with four elements, that is, $\imath^{2}=1+\imath$. The map $\bar{x}:=x^{2}$ is the unique involution on $\mathbb{F}_{4}$, which is not the identity map, i.e. $\overline{x+y}=\bar{x}+\bar{y}$, 


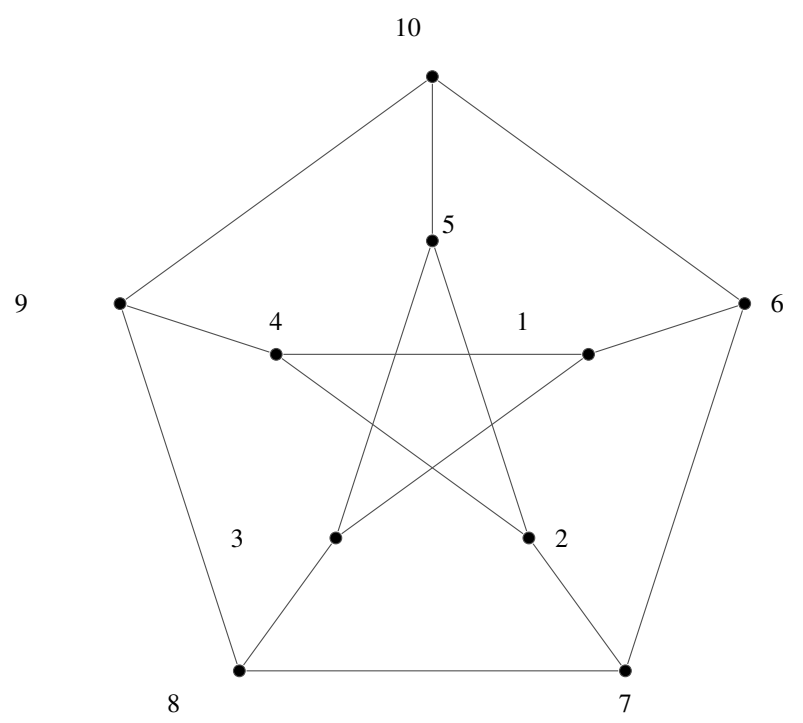

Figure 1: Graph $H G L_{2}\left(\mathbb{F}_{4}\right)$ is the Petersen graph.

$\overline{x y}=\bar{y} \cdot \bar{x}$, and $\overline{\bar{x}}=x$. Maps $\operatorname{Tr}(x):=x+\bar{x}$ and $\mathrm{N}(x):=x \bar{x}$ on $\mathbb{F}_{4}$ have $\{0,1\}$ as their image. Their preimages satisfy $\operatorname{Tr}^{-1}(0)=\{0,1\}, \operatorname{Tr}^{-1}(1)=\{\imath, 1+\imath\}, \mathrm{N}^{-1}(0)=\{0\}$, and $\mathrm{N}^{-1}(1)=\{1, \imath, 1+\imath\}$. A $n \times n$ matrix $A$ with coefficients in $\mathbb{F}_{4}$ is hermitian, if $A^{*}:=\bar{A}^{\top}=A$. Here, ${ }^{-}$is applied entry-wise on $A$, and $B^{\top}$ is the transpose of $B$. If the rank rk $A$ of a hermitian matrix equals $r$, then there is an invertible matrix $P \in G L_{n}\left(\mathbb{F}_{4}\right)$ such that

$$
A=P \operatorname{diag}(\underbrace{1, \ldots, 1}_{r}, 0 \ldots, 0) P^{*}
$$

$\left[3\right.$, Theorem 4.1], that is, $A=\sum_{j=1}^{r} \mathbf{x}_{j} \mathbf{x}_{j}^{*}$, where $\mathbf{x}_{j} \in \mathbb{F}_{4}^{n}$ is $j$-th column of matrix $P$.

Let $\mathbb{F}_{2}=\{0,1\}$ be the binary field (sometimes we will consider $\mathbb{F}_{2}$ as a subfield in $\mathbb{F}_{4}$ ) and let $A$ be a $n \times n$ matrix with coefficients in $\mathbb{F}_{2}$. Then $A$ is symmetric if $A^{\top}=A$. $\mathrm{A}$ symmetric matrix is alternate if $\mathbf{x}^{\top} A \mathbf{x}=0$ for all $\mathbf{x} \in \mathbb{F}_{2}^{n}$, or equivalently, the diagonal of $A$ is zero. If $A$, of rank $r$, is symmetric and not alternate, then there exists an invertible matrix $P \in G L_{n}\left(\mathbb{F}_{2}\right)$ such that

$$
A=P \operatorname{diag}(\underbrace{1, \ldots, 1}_{r}, 0 \ldots, 0) P^{\top}
$$

[21, Propostion 1.35], that is, $A=\sum_{j=1}^{r} \mathbf{x}_{j} \mathbf{x}_{j}^{\top}$, where $\mathbf{x}_{j} \in \mathbb{F}_{2}^{n}$ is $j$-th column of matrix $P$. If a symmetric matrix $A$ is alternate, then $\operatorname{rk} A=r$ is even and there is an invertible matrix $P$ such that

$$
A=P\left(\sum_{j=1}^{r / 2}\left(E_{2 j-1,2 j}+E_{2 j, 2 j-1}\right)\right) P^{\top},
$$

where $E_{j k}$ is the matrix with 1 at $(j, k)$-th entry and zeros elsewhere [21, Proposition 1.34]. 


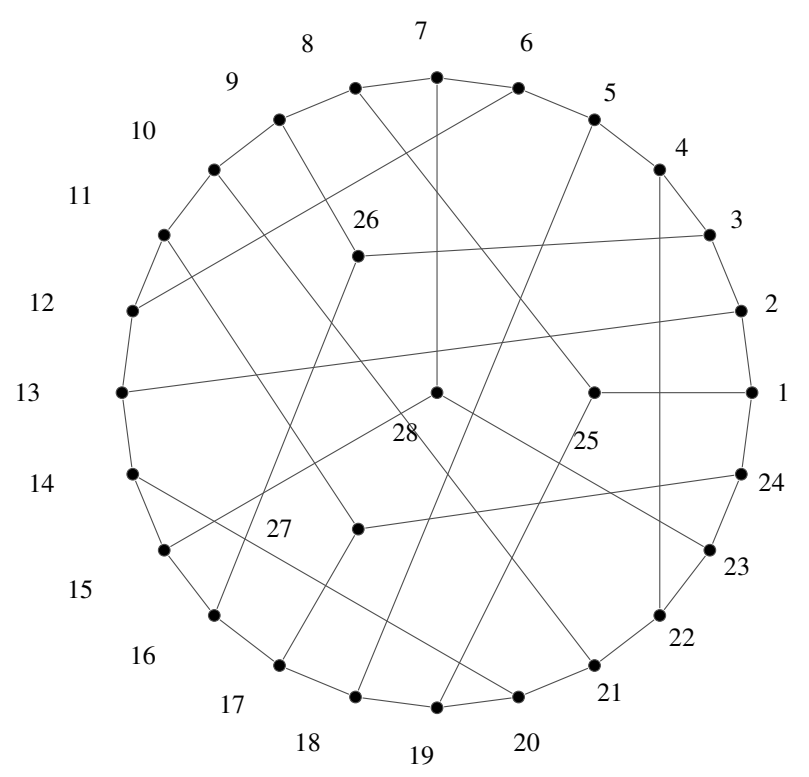

Figure 2: Graph $S G L_{3}\left(\mathbb{F}_{2}\right)$ is the Coxeter graph.

Let

$$
H_{n}\left(\mathbb{F}_{4}\right), H G L_{n}\left(\mathbb{F}_{4}\right), S_{n}\left(\mathbb{F}_{2}\right), S G L_{n}\left(\mathbb{F}_{2}\right)
$$

denote, in the same order, the sets of all hermitian $n \times n$ matrices over $\mathbb{F}_{4}$, invertible hermitian $n \times n$ matrices over $\mathbb{F}_{4}$, symmetric $n \times n$ matrices over $\mathbb{F}_{2}$, and invertible symmetric $n \times n$ matrices over $\mathbb{F}_{2}$. Each of these four sets is a vertex set of a graph, where

$$
\{A, B\} \text { is an edge if and only if } \operatorname{rk}(A-B)=1 \text {. }
$$

We slightly abuse the notation and denote these graphs with the same symbols (2). Since any symmetric matrix over $\mathbb{F}_{2}$ is also hermitian over $\mathbb{F}_{4}$, it follows that

$$
S G L_{n}\left(\mathbb{F}_{2}\right) \leqslant H G L_{n}\left(\mathbb{F}_{4}\right) \leqslant H_{n}\left(\mathbb{F}_{4}\right) \text { and } S G L_{n}\left(\mathbb{F}_{2}\right) \leqslant S_{n}\left(\mathbb{F}_{2}\right) \leqslant H_{n}\left(\mathbb{F}_{4}\right),
$$

where $\Gamma^{\prime} \leqslant \Gamma$ means that $\Gamma^{\prime}$ is an induced subgraph in $\Gamma$. Matrices $A$ and $B$ that satisfy (3) are adjacent. It is well known that the clique numbers of $H_{n}\left(\mathbb{F}_{4}\right)$ and $S_{n}\left(\mathbb{F}_{2}\right)$ both equal 2 (cf. [21, Propositions 5.7 and 6.7 ]), so these two graphs are without triangles. By (4), $H G L_{n}\left(\mathbb{F}_{4}\right)$ and $S G L_{n}\left(\mathbb{F}_{2}\right)$ are without triangles as well. Graphs $H G L_{2}\left(\mathbb{F}_{4}\right)$ and $S G L_{3}\left(\mathbb{F}_{2}\right)$ are the Petersen and the Coxeter graph, respectively, as it can be seen in Figures 1 and 2. If $k \leqslant n$ and $I_{n-k}$ is the $(n-k) \times(n-k)$ identity matrix, then the map $A \mapsto A \oplus I_{n-k}$ represents both, a graph monomorphism $H G L_{k}\left(\mathbb{F}_{4}\right) \rightarrow H G L_{n}\left(\mathbb{F}_{4}\right)$ and a graph monomorphism $S G L_{k}\left(\mathbb{F}_{2}\right) \rightarrow S G L_{n}\left(\mathbb{F}_{2}\right)$. In particular, for any $n \geqslant 2$ and $m \geqslant 3$, graphs $H G L_{n}\left(\mathbb{F}_{4}\right)$ and $S G L_{m}\left(\mathbb{F}_{2}\right)$ contain, as a subgraph, the Petersen and the Coxeter graph, respectively. The present author is not aware if the two representations of Petersen and Coxeter graph are already known or how well are they known. The representation of the Petersen graph is implicitly described in [2, p. 364], where it is mentioned that any two vertices of $H G L_{3}\left(\mathbb{F}_{4}\right)$ at distance two determine a Petersen graph. We were not able to find 
the present representation of the Coxeter graph in the literature. Some special attention to the graph $S_{3}\left(\mathbb{F}_{2}\right)$ formed by all (singular and invertible) $3 \times 3$ symmetric matrices was given in [22]. Some geometric properties of the set (not the graph) $S G L_{3}\left(\mathbb{F}_{2}\right) \backslash\{I\}$, where $I$ is the identity matrix, are described in [1].

The proofs of the main results in this paper rely on the following lemma. Recall that an $s$-arc is a sequence of vertices $\left(v_{0}, \ldots, v_{s}\right)$ such that consecutive vertices are adjacent and $v_{j-1} \neq v_{j+1}$ when $0<j<s$. An 1 -arc is simply an arc.

Lemma 1. [7, Lemma 6.9.1] Let $\Gamma$ be a connected nonbipartite graph. If every 2-arc lies in a shortest odd cycle of $\Gamma$, then $\Gamma$ is a core.

The following results give an evaluation of the determinant/inverse of a rank-one perturbation of an invertible matrix.

Lemma 2. (cf. [19]) Let $\mathbb{F}$ be a field, $A \in G L_{n}(\mathbb{F})$, and $\mathbf{x}, \mathbf{y} \in \mathbb{F}^{n}$. Then

$$
\operatorname{det}\left(A+\mathbf{x y}^{\top}\right)=(\operatorname{det} A) \cdot\left(1+\mathbf{y}^{\top} A^{-1} \mathbf{x}\right) .
$$

Corollary 3. If $A \in H G L_{n}\left(\mathbb{F}_{4}\right)$ and $\mathbf{x} \in \mathbb{F}_{4}^{n}$, then $A+\mathbf{x x}^{*}$ is invertible iff $\mathbf{x}^{*} A^{-1} \mathbf{x}=0$.

Proof. Since $\mathbf{x}^{*} A^{-1} \mathbf{x} \in \mathbb{F}_{2}$, the result follows from Lemma 2 if we choose $\mathbf{y}:=\overline{\mathbf{x}}$.

Corollary 4. If $A \in S G L_{n}\left(\mathbb{F}_{2}\right)$ and $\mathbf{x} \in \mathbb{F}_{2}^{n}$, then $A+\mathbf{x x}^{\top}$ is invertible iff $\mathbf{x}^{\top} A^{-1} \mathbf{x}=0$.

Proof. Since $\mathbf{x}^{\top} A^{-1} \mathbf{x} \in \mathbb{F}_{2}$, the result follows from Lemma 2 if we choose $\mathbf{y}:=\mathbf{x}$.

Corollary 5. Suppose that $A, A+\mathbf{x x}^{*} \in H G L_{n}\left(\mathbb{F}_{4}\right)$ for some $\mathbf{x} \in \mathbb{F}_{4}^{n}$. Then

$$
\left(A+\mathbf{x x}^{*}\right)^{-1}=A^{-1}+\left(A^{-1} \mathbf{x}\right)\left(A^{-1} \mathbf{x}\right)^{*} .
$$

Proof. By Corollary $3, \mathbf{x}^{*} A^{-1} \mathbf{x}=0$. Since $\left(A^{-1}\right)^{*}=A^{-1}$, it follows that

$$
\begin{aligned}
\left(A+\mathbf{x x}^{*}\right) & \left(A^{-1}+\left(A^{-1} \mathbf{x}\right)\left(A^{-1} \mathbf{x}\right)^{*}\right)= \\
& =I+\mathbf{x x}^{*} A^{-1}+\mathbf{x x}^{*} A^{-1}+\mathbf{x}\left(\mathbf{x}^{*} A^{-1} \mathbf{x}\right)\left(A^{-1} \mathbf{x}\right)^{*}=I .
\end{aligned}
$$

Corollary 6. Suppose that $A, A+\mathbf{x x}^{\top} \in S G L_{n}\left(\mathbb{F}_{2}\right)$ for some $\mathbf{x} \in \mathbb{F}_{2}^{n}$. Then

$$
\left(A+\mathbf{x x}^{\top}\right)^{-1}=A^{-1}+\left(A^{-1} \mathbf{x}\right)\left(A^{-1} \mathbf{x}\right)^{\top} .
$$

Proof. Replace $\mathbf{x}^{*}$ by $\mathbf{x}^{\top}$ and Corollary 3 by Corollary 4 in the proof of Corollary 5 . 


\section{Main results}

We now state the main results of this paper.

Theorem 7. $H G L_{n}\left(\mathbb{F}_{4}\right)$ is a core for $n \geqslant 2$.

Theorem 8. $S G L_{n}\left(\mathbb{F}_{2}\right)$ is a core $n \geqslant 3$.

Remark 9. Graph $S G L_{2}\left(\mathbb{F}_{2}\right)$ is just a star on four vertices, so it is not a core, since there exists an obvious endomorphism with a path of distance two or one as its image.

To prove Theorems 7 and 8 we need few more lemmas. The proof of Lemma 10 and some of the proofs in the sequel are written (partially) simultaneously for $H G L_{n}\left(\mathbb{F}_{4}\right)$ and $S G L_{n}\left(\mathbb{F}_{2}\right)$. In these cases $\left(A^{\dagger}, \tau\right)$ denotes $\left(A^{*},^{-}\right)$and $\left(A^{\top}\right.$, identity map), when considering hermitian and symmetric matrices, respectively.

Lemma 10. Graphs $H G L_{n}\left(\mathbb{F}_{4}\right)$ and $S G L_{n}\left(\mathbb{F}_{2}\right)$ are connected for $n \geqslant 2$.

Proof. If $A \in S G L_{n}\left(\mathbb{F}_{2}\right)$ is alternate, then there exists $B \in S G L_{n}\left(\mathbb{F}_{2}\right)$, which is not alternate and is adjacent to $A$. In fact, if $A$ is of the form (1), then $B:=A+P E_{11} P^{\top}$ has the mentioned properties. So for graph $S G L_{n}\left(\mathbb{F}_{2}\right)$ it suffices to find a walk between symmetric matrices that are not alternate. More generally, for $\Gamma \in\left\{H G L_{n}\left(\mathbb{F}_{4}\right), S G L_{n}\left(\mathbb{F}_{2}\right)\right\}$ we need to find a walk in $\Gamma$ between any $A=\sum_{j=1}^{n} \mathbf{x}_{j} \mathbf{x}_{j}^{\dagger}$ and $B=\sum_{j=1}^{n} \mathbf{y}_{j} \mathbf{y}_{j}^{\dagger}$ that are invertible. We split the proof in two steps.

Step 1. If $A=\sum_{j=1}^{n} \mathbf{x}_{j} \mathbf{x}_{j}^{\dagger}$ and $B=\mathbf{y}_{1} \mathbf{y}_{1}^{\dagger}+\sum_{j=2}^{n} \mathbf{x}_{j} \mathbf{x}_{j}^{\dagger}$ are invertible, then there exists a walk in $\Gamma$ from $A$ to $B$.

We may assume that $A \neq B$. Choose the invertible matrix $P$ with $\mathbf{x}_{j}$ as the $j$-th column, that is, $\mathbf{x}_{j}=P \mathbf{e}_{j}$ for all $j$, where $\mathbf{e}_{j}=(0, \ldots, 0,1,0, \ldots, 0)^{\top}$ is the $j$-th vector of the standard basis. Denote $\left(z_{1}, \ldots, z_{n}\right)^{\top}=\mathbf{z}:=P^{-1} \mathbf{y}_{1}$. Since $C:=P^{-1} A\left(P^{-1}\right)^{\dagger}=I$ and $D:=P^{-1} B\left(P^{-1}\right)^{\dagger}=E_{22}+\ldots+E_{n n}+\mathbf{z z}^{\dagger}$ are invertible and distinct, it follows that $z_{1} \neq 0$ and $z_{k} \neq 0$ for some $k \geqslant 2$. Consequently, $z_{1} z_{1}^{\tau}=1=z_{k} z_{k}^{\tau}$.

If $\mathbf{z}^{\dagger} \mathbf{z}=0$, then $I+\mathbf{z z}^{\dagger}$ is invertible by Corollary $3 /$ Corollary 4 , so $C, I+\mathbf{z z}^{\dagger}, D$ is a walk from $C$ to $D$ and $A, P\left(I+\mathbf{z z}^{\dagger}\right) P^{\dagger}, B$ is a walk from $A$ to $B$.

Assume now that $\mathbf{z}^{\dagger} \mathbf{z} \neq 0$, i.e., $\mathbf{z}^{\dagger} \mathbf{z}=1$. Matrices $A_{1}:=I+\left(z_{1} \mathbf{e}_{1}+z_{k} \mathbf{e}_{k}\right)\left(z_{1} \mathbf{e}_{1}+z_{k} \mathbf{e}_{k}\right)^{\dagger}$ and $A_{2}:=I+\left(z_{1} \mathbf{e}_{1}+z_{k} \mathbf{e}_{k}\right)\left(z_{1} \mathbf{e}_{1}+z_{k} \mathbf{e}_{k}\right)^{\dagger}+E_{11}$ are invertible. Moreover,

$$
\begin{aligned}
\mathbf{z}^{\dagger}\left(I+\left(z_{1} \mathbf{e}_{1}+z_{k} \mathbf{e}_{k}\right)\right. & \left.\left(z_{1} \mathbf{e}_{1}+z_{k} \mathbf{e}_{k}\right)^{\dagger}+E_{11}\right)^{-1} \mathbf{z} \\
& =\mathbf{z}^{\dagger}\left(I+\left(z_{1} \mathbf{e}_{1}+z_{k} \mathbf{e}_{k}\right)\left(z_{1} \mathbf{e}_{1}+z_{k} \mathbf{e}_{k}\right)^{\dagger}+E_{k k}\right) \mathbf{z} \\
& =\mathbf{z}^{\dagger} \mathbf{z}+\left(z_{1} z_{1}^{\tau}+z_{k} z_{k}^{\tau}\right)^{2}+z_{k} z_{k}^{\tau}=0,
\end{aligned}
$$

so $A_{3}:=I+\left(z_{1} \mathbf{e}_{1}+z_{k} \mathbf{e}_{k}\right)\left(z_{1} \mathbf{e}_{1}+z_{k} \mathbf{e}_{k}\right)^{\dagger}+E_{11}+\mathbf{z z}^{\dagger}$ is invertible and $C, A_{1}, A_{2}, A_{3}, D$ is a walk from $C$ to $D$. Consequently, $A, P A_{1} P^{\dagger}, P A_{2} P^{\dagger}, P A_{3} P^{\dagger}, B$ is a walk from $A$ to $B$. 
Step 2. If $A=\sum_{j=1}^{n} \mathbf{x}_{j} \mathbf{x}_{j}^{\dagger}$ and $B=\sum_{j=1}^{n} \mathbf{y}_{j} \mathbf{y}_{j}^{\dagger}$ are invertible, then there exists a walk in $\Gamma$ from $A$ to $B$, so $\Gamma$ is connected.

Denote $A_{1}:=A$. Obviously, $\mathbf{x}_{1}, \ldots, \mathbf{x}_{n}$ are linearly independent. The same holds for $\mathbf{y}_{1}, \ldots, \mathbf{y}_{n}$. There exists $j_{1}$ such that $\mathbf{y}_{j_{1}}$ is not in the linear $\operatorname{span} \operatorname{Lin}\left\{\mathbf{x}_{2}, \ldots, \mathbf{x}_{n}\right\}$. Then $\mathbf{y}_{j_{1}}, \mathbf{x}_{2}, \ldots, \mathbf{x}_{n}$ are linearly independent, so $A_{2}:=\mathbf{y}_{j_{1}} \mathbf{y}_{j_{1}}^{\dagger}+\sum_{j=2}^{n} \mathbf{x}_{j} \mathbf{x}_{j}^{\dagger}$ is invertible. There exists $j_{2}$ such that $\mathbf{y}_{j_{2}} \notin \operatorname{Lin}\left\{\mathbf{y}_{j_{1}}, \mathbf{x}_{3}, \ldots, \mathbf{x}_{n}\right\}$. Obvioulsly $j_{2} \neq j_{1}$. Consequently, $\mathbf{y}_{j_{1}}, \mathbf{y}_{j_{2}}, \mathbf{x}_{3}, \ldots, \mathbf{x}_{n}$ are linearly independent, so $A_{3}:=\mathbf{y}_{j_{1}} \mathbf{y}_{j_{1}}^{\dagger}+\mathbf{y}_{j_{2}} \mathbf{y}_{j_{2}}^{\dagger}+\sum_{j=3}^{n} \mathbf{x}_{j} \mathbf{x}_{j}^{\dagger}$ is invertible. There exists $j_{3}$ such that $\mathbf{y}_{j_{3}} \notin \operatorname{Lin}\left\{\mathbf{y}_{j_{1}}, \mathbf{y}_{j_{2}}, \mathbf{x}_{4}, \ldots, \mathbf{x}_{n}\right\}$. Obvioulsly $j_{3} \neq j_{1}, j_{2}$. Consequently, $\mathbf{y}_{j_{1}}, \mathbf{y}_{j_{2}}, \mathbf{y}_{j_{3}}, \mathbf{x}_{4}, \ldots, \mathbf{x}_{n}$ are linearly independent, so $A_{4}:=\mathbf{y}_{j_{1}} \mathbf{y}_{j_{1}}^{\dagger}+\mathbf{y}_{j_{2}} \mathbf{y}_{j_{2}}^{\dagger}+$ $\mathbf{y}_{j_{3}} \mathbf{y}_{j_{3}}^{\dagger}+\sum_{j=4}^{n} \mathbf{x}_{j} \mathbf{x}_{j}^{\dagger}$ is invertible. We continue in the same way and obtain invertible matrices $A_{5}, \ldots, A_{n+1}=B$ with $A_{k}=\sum_{i=1}^{k-1} \mathbf{y}_{j_{i}} \mathbf{y}_{j_{i}}^{\dagger}+\sum_{j=k}^{n} \mathbf{x}_{j} \mathbf{x}_{j}^{\dagger}$. By Step 1 there exists a walk from $A_{k}$ to $A_{k+1}$ for each $k$, so we obtain a walk from $A=A_{1}$ to $B=A_{n+1}$.

We now provide a proof of the first main result. In Section 4 we present a shorter but more involved proof, which relies on additional results.

Proof of Theorem \%. Recall from preliminaries that $H G L_{n}\left(\mathbb{F}_{4}\right)$ has no triangles. By Lemma 10 it is connected. We will show that any 2-arc in $H G L_{n}\left(\mathbb{F}_{4}\right)$ lies in a 5-cycle. This will end the proof by Lemma 1, since a graph with a 5-cycle has the chromatic number at least three and it is therefore nonbipartite.

Assume that $B_{1}, B_{2}, B_{3}$ is a 2 -arc in $H G L_{n}\left(\mathbb{F}_{4}\right)$, that is, $B_{j}$ is invertible with $\operatorname{rk}\left(B_{j}-\right.$ $\left.B_{j+1}\right)=1$ and $B_{3} \neq B_{1}$. Then there exist two nonzero column vectors $\mathbf{x}$ and $\mathbf{y}$ such that $B_{2}=B_{1}+\mathbf{x x}^{*}$ and $B_{3}=B_{1}+\mathbf{x x}^{*}+\mathbf{y y}^{*}$. Vectors $\mathbf{x}$ and $\mathbf{y}$ are linearly independent, since the contrary would imply that $\mathbf{x x}^{*}=\mathbf{y y}^{*}$, i.e., $B_{3}=B_{1}$. Consequently, there is an invertible matrix $P$ such that $P \mathbf{e}_{1}=\mathbf{x}$ and $P \mathbf{e}_{2}=\mathbf{y}$. Let $A:=P^{-1} B_{1}\left(P^{-1}\right)^{*}$. We will first show that the 2-arc $A, A+E_{11}=P^{-1} B_{2}\left(P^{-1}\right)^{*}, A+E_{11}+E_{22}=P^{-1} B_{3}\left(P^{-1}\right)^{*}$ lies in a 5 -cycle.

Let $c_{j k}$ denote the $(j, k)$-th entry of the inverse $A^{-1}$. Then

$$
c_{k j}=\overline{c_{j k}} .
$$

Since $A+E_{11}=A+\mathbf{e}_{1} \mathbf{e}_{1}^{*}$ is invertible, it follows from Corollary 3 that

$$
0=\mathbf{e}_{1}^{*} A^{-1} \mathbf{e}_{1}=c_{11} .
$$

Since $A+E_{11}+E_{22}$ is invertible, it follows from Corollary 3, Corollary 5, and (5) that

$$
0=\mathbf{e}_{2}^{*}\left(A+E_{11}\right)^{-1} \mathbf{e}_{2}=\mathbf{e}_{2}^{*}\left(A^{-1}+\left(A^{-1} \mathbf{e}_{1}\right)\left(A^{-1} \mathbf{e}_{1}\right)^{*}\right) \mathbf{e}_{2}=c_{22}+c_{12} \overline{c_{12}} .
$$

From preliminaries we know that there exists a nonzero $a \in \mathbb{F}_{4}$ such that

$$
\mathrm{N}\left(c_{12}\right)=\operatorname{Tr}\left(a \overline{c_{12}}\right)
$$

From (6)-(8) we deduce that

$$
\left(\mathbf{e}_{1}+\bar{a} \mathbf{e}_{2}\right)^{*} A^{-1}\left(\mathbf{e}_{1}+\bar{a} \mathbf{e}_{2}\right)=c_{12} \overline{c_{12}}+a \overline{c_{12}}+\bar{a} c_{12}=0,
$$


so matrix $A+E_{11}+a E_{12}+\bar{a} E_{21}+E_{22}=A+\left(\mathbf{e}_{1}+\bar{a} \mathbf{e}_{2}\right)\left(\mathbf{e}_{1}+\bar{a} \mathbf{e}_{2}\right)^{*}$ is invertible. Define

$$
b:=\left\{\begin{array}{ll}
a\left(a \overline{c_{12}}-1\right) & \text { if } c_{12} \neq 0 \\
\imath a & \text { if } c_{12}=0
\end{array} .\right.
$$

Note that $a \neq b$. Moreover, (8) implies that $b \neq 0$. Recall that $\mathrm{N}(x)=1$ for all nonzero $x \in \mathbb{F}_{4}$. Hence, Corollary 5, a straightforward computation, and (6)-(10) show that

$$
\begin{aligned}
& \left(\mathbf{e}_{1}+\bar{b} \mathbf{e}_{2}\right)^{*}\left(A+\left(\mathbf{e}_{1}+\bar{a} \mathbf{e}_{2}\right)\left(\mathbf{e}_{1}+\bar{a} \mathbf{e}_{2}\right)^{*}\right)^{-1}\left(\mathbf{e}_{1}+\bar{b} \mathbf{e}_{2}\right)= \\
& =\left(\mathbf{e}_{1}+\bar{b} \mathbf{e}_{2}\right)^{*}\left(A^{-1}+\left(A^{-1}\left(\mathbf{e}_{1}+\bar{a} \mathbf{e}_{2}\right)\right)\left(A^{-1}\left(\mathbf{e}_{1}+\bar{a} \mathbf{e}_{2}\right)\right)^{*}\right)\left(\mathbf{e}_{1}+\bar{b} \mathbf{e}_{2}\right)=0
\end{aligned}
$$

SO

$$
A+\left(\mathbf{e}_{1}+\bar{a} \mathbf{e}_{2}\right)\left(\mathbf{e}_{1}+\bar{a} \mathbf{e}_{2}\right)^{*}+\left(\mathbf{e}_{1}+\bar{b} \mathbf{e}_{2}\right)\left(\mathbf{e}_{1}+\bar{b} \mathbf{e}_{2}\right)^{*}=A+(a+b) E_{12}+\overline{(a+b)} E_{21}
$$

is invertible with $a+b \neq 0$. Hence, $A, A+E_{11}, A+E_{11}+E_{22}, A+(a+b) E_{12}+\overline{(a+b)} E_{21}$, $A+E_{11}+a E_{12}+\bar{a} E_{21}+E_{22}, A$ is a 5-cycle. Since $\operatorname{rk}\left(P X P^{*}\right)=\operatorname{rk} X$ for all $X$, the map $X \mapsto P X P^{*}$ is an automorphism of graph $H G L_{n}\left(\mathbb{F}_{4}\right)$, so $B_{1}, B_{2}, B_{3}, P\left(A+(a+b) E_{12}+\right.$ $\left.\overline{(a+b)} E_{21}\right) P^{*}, P\left(A+E_{11}+a E_{12}+\bar{a} E_{21}+E_{22}\right) P^{*}, B_{1}$ is a 5 -cycle as well.

To prove Theorem 8 we need an additional technical lemma.

Lemma 11. Let $n \geqslant 3$.

(i) If $A \in S G L_{n}\left(\mathbb{F}_{2}\right)$, then $\left|\left\{\mathbf{x} \in \mathbb{F}_{2}^{n} \backslash\{0\}: \mathbf{x}^{\top} A \mathbf{x}=0\right\}\right| \geqslant 3$. Equality holds iff $n=3$.

(ii) If $A, A+\mathbf{x} \mathbf{x}^{\top}, A+\mathbf{x} \mathbf{x}^{\top}+\mathbf{y y}^{\top} \in S G L_{n}\left(\mathbb{F}_{2}\right)$ are distinct, then there is $\mathbf{z} \in \mathbb{F}_{2}^{n}$ such that $\mathbf{x}, \mathbf{y}, \mathbf{z}$ are linearly independent and $A+\mathbf{x} \mathbf{x}^{\top}+\mathbf{y} \mathbf{y}^{\top}+\mathbf{z} \mathbf{z}^{\top} \in S G L_{n}\left(\mathbb{F}_{2}\right)$.

Proof. (i) If $A$ is alternate, then $n$ is even and $\mathbf{x}^{\top} A \mathbf{x}=0$ for all $\mathbf{x}$. If $A$ is not alternate, then $A=P P^{\top}$ for some invertible matrix $P$, so $\mathbf{x} \mapsto\left(P^{\top}\right)^{-1} \mathbf{x}$ is a bijection between the sets $\left\{\mathbf{x} \in \mathbb{F}_{2}^{n} \backslash\{0\}: \mathbf{x}^{\top} \mathbf{x}=0\right\}$ and $\left\{\mathbf{x} \in \mathbb{F}_{2}^{n} \backslash\{0\}: \mathbf{x}^{\top} A \mathbf{x}=0\right\}$. Since $\mathbf{x}^{\top} \mathbf{x}=0$ iff even number of coordinates of $\mathbf{x}$ equal 1 , we deduce that

$$
\left|\left\{\mathbf{x} \in \mathbb{F}_{2}^{n} \backslash\{0\}: \mathbf{x}^{\top} A \mathbf{x}=0\right\}\right|=\sum_{k=1}^{\lfloor n / 2\rfloor}\left(\begin{array}{c}
n \\
2 k
\end{array}\right) \geqslant 3
$$

with equality iff $n=3$.

(ii) The assumptions imply that $\mathbf{x}$ and $\mathbf{y}$ are linearly independent. Let $B:=A+\mathbf{x} \mathbf{x}^{\top}+$ $\mathbf{y y}^{\top}$. If $n \geqslant 4$, then $\left|\left\{\mathbf{z} \in \mathbb{F}_{2}^{n} \backslash\{0\}: \mathbf{z}^{\top} B^{-1} \mathbf{z}=0\right\}\right|>3$ by (i). Since $\operatorname{Lin}\{\mathbf{x}, \mathbf{y}\} \backslash\{0\}=$ $\{\mathbf{x}, \mathbf{y}, \mathbf{x}+\mathbf{y}\}$, there is $\mathbf{z} \notin \operatorname{Lin}\{\mathbf{x}, \mathbf{y}\}$ such that $\mathbf{z}^{\top} B^{-1} \mathbf{z}=0$. By Corollary $4, B+\mathbf{z z}^{\top}$ is invertible, so the proof ends. Let $n=3$, so $\left|\left\{\mathbf{z} \in \mathbb{F}_{2}^{n} \backslash\{0\}: \mathbf{z}^{\top} B^{-1} \mathbf{z}=0\right\}\right|=3$. Assume for a contradiction that there is no $\mathbf{z} \notin \operatorname{Lin}\{\mathbf{x}, \mathbf{y}\}$ such that $\mathbf{z}^{\top} B^{-1} \mathbf{z}=0$, that is,

$$
\left\{\mathbf{z} \in \mathbb{F}_{2}^{n} \backslash\{0\}: \mathbf{z}^{\top} B^{-1} \mathbf{z}=0\right\}=\{\mathbf{x}, \mathbf{y}, \mathbf{x}+\mathbf{y}\}
$$


Since $A+\mathbf{x x}^{\top}$ and $A+\mathbf{x} \mathbf{x}^{\top}+\mathbf{y y}^{\top}$ are invertible, Corollaries 4 and 6 imply that

$$
0=\mathbf{x}^{\top} A^{-1} \mathbf{x}
$$

and

$$
0=\mathbf{y}^{\top}\left(A+\mathbf{x} \mathbf{x}^{\top}\right)^{-1} \mathbf{y}=\mathbf{y}^{\top} A^{-1} \mathbf{y}+\mathbf{y}^{\top}\left(\left(A^{-1} \mathbf{x}\right)\left(A^{-1} \mathbf{x}\right)^{\top}\right) \mathbf{y}=\mathbf{y}^{\top} A^{-1} \mathbf{y}+\mathbf{y}^{\top} A^{-1} \mathbf{x} .
$$

Corollary 6 shows that

$$
\begin{aligned}
B^{-1} & =\left(A+\mathbf{x} \mathbf{x}^{\top}\right)^{-1}+\left(\left(A+\mathbf{x} \mathbf{x}^{\top}\right)^{-1} \mathbf{y}\right)\left(\left(A+\mathbf{x} \mathbf{x}^{\top}\right)^{-1} \mathbf{y}\right)^{\top} \\
& =A^{-1}+\left(A^{-1} \mathbf{x}\right)\left(A^{-1} \mathbf{x}\right)^{\top}+\left(A^{-1} \mathbf{y}+A^{-1} \mathbf{x} \mathbf{x}^{\top} A^{-1} \mathbf{y}\right)\left(A^{-1} \mathbf{y}+A^{-1} \mathbf{x} \mathbf{x}^{\top} A^{-1} \mathbf{y}\right)^{\top} .
\end{aligned}
$$

Since $\mathbf{x}^{\top} B^{-1} \mathbf{x}=0$ by (12), equations (15), (13), and (14) imply that

$$
\mathbf{y}^{\top} A^{-1} \mathbf{x}=0=\mathbf{y}^{\top} A^{-1} \mathbf{y} .
$$

Choose $Q$ such that $A^{-1}=Q Q^{\top}$. If $\mathbf{u}:=Q^{\top} \mathbf{x}$ and $\mathbf{v}:=Q^{\top} \mathbf{y}$, then (13) and (16) force

$$
0=\mathbf{u}^{\top} \mathbf{u}=\mathbf{v}^{\top} \mathbf{v}=(\mathbf{u}+\mathbf{v})^{\top}(\mathbf{u}+\mathbf{v}) \text { and } 0=\mathbf{u}^{\top} \mathbf{v} .
$$

The first three equations of (17) shows that $\{\mathbf{u}, \mathbf{v}, \mathbf{u}+\mathbf{v}\}=\left\{(1,1,0)^{\top},(1,0,1)^{\top},(0,1,1)^{\top}\right\}$, which contradicts the fourth equation in (17).

Proof of Theorem 8. From preliminaries we know that there are no triangles in $S G L_{n}\left(\mathbb{F}_{2}\right)$. We now show that neither 5 -cycles exist. Assume that $A_{1}, A_{2}, \ldots, A_{5}, A_{6}=A_{1}$ is a 5-cycle. Then $A_{j}=A_{1}+\sum_{k=1}^{j-1} \mathbf{x}_{k} \mathbf{x}_{k}^{\top}$ for some nonzero column vectors $\mathbf{x}_{1}, \ldots, \mathbf{x}_{5}$, so $\sum_{k=1}^{5} \mathbf{x}_{k} \mathbf{x}_{k}^{\top}=0$. Consequently,

$$
\mathbf{x}_{1} \mathbf{x}_{1}^{\top}+\mathbf{x}_{2} \mathbf{x}_{2}^{\top}=\mathbf{x}_{3} \mathbf{x}_{3}^{\top}+\mathbf{x}_{4} \mathbf{x}_{4}^{\top}+\mathbf{x}_{5} \mathbf{x}_{5}^{\top} .
$$

Since $A_{3} \neq A_{1}$, we deduce that $\mathbf{x}_{1} \neq \mathbf{x}_{2}$ and the matrix in (18) is of rank two. Therefore $\mathbf{x}_{3}, \mathbf{x}_{4}, \mathbf{x}_{5}$ are all distinct and span a 2-dimension vector subspace in $\mathbb{F}_{2}^{n}$, that is, $\mathbf{x}_{5}=$ $\mathbf{x}_{3}+\mathbf{x}_{4}$, so the matrix on the right side of equation (18) equals $\mathbf{x}_{3} \mathbf{x}_{4}^{\top}+\mathbf{x}_{4} \mathbf{x}_{3}^{\top}$, which is an alternate matrix. We are in contradiction, since the matrix on the left side is not alternate.

The proof will end by Lemma 1 and Lemma 10 if we show that any 2 -arc $A, A+\mathbf{x x}^{\top}$, $A+\mathbf{x} \mathbf{x}^{\top}+\mathbf{y y}^{\top}$ lies in a 7 -cycle. By Lemma 11 there is $\mathbf{z}$ such that $\mathbf{x}, \mathbf{y}, \mathbf{z}$ are linearly independent and $A+\mathbf{x} \mathbf{x}^{\top}+\mathbf{y} \mathbf{y}^{\top}+\mathbf{z z}^{\top}$ is invertible. Pick an invertible matrix $P$ such that $\mathbf{x}=P \mathbf{e}_{1}, \mathbf{y}=P \mathbf{e}_{2}$, and $\mathbf{z}=P \mathbf{e}_{3}$ and let $B:=P^{-1} A\left(P^{-1}\right)^{\top}$. Since the map $X \mapsto P X P^{\top}$ is an automorphism of $S G L_{n}\left(\mathbb{F}_{2}\right)$, it suffices to show that the 3 -arc

$$
B, B+\mathbf{e}_{1} \mathbf{e}_{1}^{\top}, B+\mathbf{e}_{1} \mathbf{e}_{1}^{\top}+\mathbf{e}_{2} \mathbf{e}_{2}^{\top}, B+\mathbf{e}_{1} \mathbf{e}_{1}^{\top}+\mathbf{e}_{2} \mathbf{e}_{2}^{\top}+\mathbf{e}_{3} \mathbf{e}_{3}^{\top}
$$

lies in a 7 -cycle. Let $b_{j k}$ denote the $(j, k)$-th entry of the inverse $B^{-1}$. Then $b_{j k}=b_{k j}$. Since matrices in (19) are invertible, Corollary 4 shows that

$$
0=\mathbf{e}_{1} B^{-1} \mathbf{e}_{1}=b_{11},
$$


equality $0=\mathbf{e}_{2}\left(B+\mathbf{e}_{1} \mathbf{e}_{1}^{\top}\right)^{-1} \mathbf{e}_{2}=b_{22}+b_{12}^{2}$ forces

$$
b_{22}=b_{12} \text {, }
$$

and

$$
\begin{aligned}
0 & =\mathbf{e}_{3}^{\top}\left(B+\mathbf{e}_{1} \mathbf{e}_{1}^{\top}+\mathbf{e}_{2} \mathbf{e}_{2}^{\top}\right)^{-1} \mathbf{e}_{3} \\
& =\mathbf{e}_{3}^{\top}\left(B+\mathbf{e}_{1} \mathbf{e}_{1}^{\top}\right)^{-1} \mathbf{e}_{3}+\mathbf{e}_{3}^{\top}\left(\left(B+\mathbf{e}_{1} \mathbf{e}_{1}^{\top}\right)^{-1} \mathbf{e}_{2}\right)\left(\left(B+\mathbf{e}_{1} \mathbf{e}_{1}^{\top}\right)^{-1} \mathbf{e}_{2}\right)^{\top} \mathbf{e}_{3} \\
& =b_{33}+b_{13}^{2}+\left(b_{23}+b_{13} b_{12}\right)^{2}
\end{aligned}
$$

forces

$$
b_{33}=b_{23}+b_{13}\left(1+b_{12}\right) .
$$

\begin{tabular}{|c|c|c|c|c|c|c|c|}
\hline$C$ & $\mathbf{x}_{1}$ & $\mathbf{x}_{2}$ & $\mathbf{x}_{3}$ & $\mathbf{x}_{4}$ & $\mathbf{x}_{5}$ & $\mathbf{x}_{6}$ & $\mathrm{x}_{7}$ \\
\hline$\left(\begin{array}{lll}0 & 1 & 1 \\
1 & 1 & 0 \\
1 & 0 & 0\end{array}\right)$ & $\mathbf{e}_{1}$ & $\mathbf{e}_{2}$ & $\mathbf{e}_{3}$ & $\mathbf{e}_{1}+\mathbf{e}_{2}$ & $\mathbf{e}_{2}+\mathbf{e}_{3}$ & $\mathbf{e}_{1}+\mathbf{e}_{2}+\mathbf{e}_{3}$ & $\mathbf{e}_{1}+\mathbf{e}_{3}$ \\
\hline$\left(\begin{array}{lll}0 & 1 & 0 \\
1 & 1 & 0 \\
0 & 0 & 0\end{array}\right)$ & $\mathbf{e}_{1}$ & $\mathbf{e}_{2}$ & $\mathbf{e}_{3}$ & $\mathbf{e}_{2}+\mathbf{e}_{3}$ & $\mathbf{e}_{1}+\mathbf{e}_{2}$ & $\mathbf{e}_{1}+\mathbf{e}_{2}+\mathbf{e}_{3}$ & $\mathbf{e}_{1}+\mathbf{e}_{3}$ \\
\hline$\left(\begin{array}{lll}0 & 1 & 1 \\
1\end{array}\right)$ & $\mathbf{e}_{1}$ & $\mathbf{e}_{2}$ & $\mathbf{e}_{3}$ & $\mathbf{e}_{2}+\mathbf{e}_{3}$ & $\mathbf{e}_{1}+\mathbf{e}_{3}$ & $\mathbf{e}_{1}+\mathbf{e}_{2}$ & $\mathbf{e}_{1}+\mathbf{e}_{2}+\mathbf{e}_{3}$ \\
\hline$\left(\begin{array}{lll}0 & 1 & 0 \\
1 & 1 & 1 \\
0 & 1 & 1\end{array}\right)$ & $\mathbf{e}_{1}$ & $\mathbf{e}_{2}$ & $\mathbf{e}_{3}$ & $\mathbf{e}_{1}+\mathbf{e}_{3}$ & $\mathbf{e}_{1}+\mathbf{e}_{2}+\mathbf{e}_{3}$ & $\mathbf{e}_{1}+\mathbf{e}_{2}$ & $\mathbf{e}_{2}+\mathbf{e}_{3}$ \\
\hline$\left(\begin{array}{lll}0 & 0 & 0 \\
0 & 0 & 1 \\
0 & 1 & 1\end{array}\right)$ & $\mathbf{e}_{1}$ & $\mathbf{e}_{2}$ & $\mathbf{e}_{3}$ & $\mathbf{e}_{1}+\mathbf{e}_{3}$ & $\mathbf{e}_{1}+\mathbf{e}_{2}+\mathbf{e}_{3}$ & $\mathbf{e}_{2}+\mathbf{e}_{3}$ & $\mathbf{e}_{1}+\mathbf{e}_{2}$ \\
\hline$\left(\begin{array}{lll}0 & 0 & 1 \\
0 & 0 & 0 \\
1 & 0 & 1\end{array}\right)$ & $\mathbf{e}_{1}$ & $\mathbf{e}_{2}$ & $\mathbf{e}_{3}$ & $\mathbf{e}_{2}+\mathbf{e}_{3}$ & $\mathbf{e}_{1}+\mathbf{e}_{2}+\mathbf{e}_{3}$ & $\mathbf{e}_{1}+\mathbf{e}_{3}$ & $\mathbf{e}_{1}+\mathbf{e}_{2}$ \\
\hline$\left(\begin{array}{lll}0 & 0 & 1 \\
0 & 0 & 1 \\
1 & 1 & 0\end{array}\right)$ & $\mathbf{e}_{1}$ & $\mathbf{e}_{2}$ & $\mathbf{e}_{3}$ & $\mathbf{e}_{1}+\mathbf{e}_{2}+\mathbf{e}_{3}$ & $\mathbf{e}_{2}+\mathbf{e}_{3}$ & $\mathbf{e}_{1}+\mathbf{e}_{3}$ & $\mathbf{e}_{1}+\mathbf{e}_{2}$ \\
\hline$\left(\begin{array}{lll}0 & 0 & 0 \\
0 & 0 & 0 \\
0 & 0 & 0\end{array}\right)$ & $\mathbf{e}_{1}$ & $\mathbf{e}_{2}$ & $\mathbf{e}_{3}$ & $\mathbf{e}_{1}+\mathbf{e}_{2}$ & $\mathbf{e}_{1}+\mathbf{e}_{3}$ & $\mathbf{e}_{2}+\mathbf{e}_{3}$ & $\mathbf{e}_{1}+\mathbf{e}_{2}+\mathbf{e}_{3}$ \\
\hline
\end{tabular}

Consequently, (20)-(22) show that there are only $2^{3}=8$ possibilities for the upper-left $3 \times 3$ block of $B^{-1}$, which we denote by $C$. We define matrices $B=B_{1}, B_{2}, \ldots, B_{7}, B_{8}$ with $B_{j}:=B_{1}+\sum_{k=1}^{j-1} \mathbf{x}_{k} \mathbf{x}_{k}^{\top}$, where vectors $\mathbf{x}_{k}$ depend on $C$ and are as in the table below

Observe that $\sum_{k=1}^{7} \mathbf{x}_{k} \mathbf{x}_{k}^{\top}=0$, so $B_{8}=B_{1}$ and it is easy to see that $B_{j} \neq B_{k}$ for $1 \leqslant j<k \leqslant 7$. Hence, to prove that $B_{1}, \ldots, B_{8}$ is a 7 -cycle with required properties we need to show that matrices $B_{j}$ are invertible. We already know that $B_{1}, B_{2}, B_{3}, B_{4}$ are such, since they form the 3 -arc (19). Since $B_{7}=B_{1}+\mathbf{x}_{7} \mathbf{x}_{7}^{\top}$ and $\mathbf{x}_{7}^{\top} B_{1}^{-1} \mathbf{x}_{7}=\mathbf{x}_{7}^{\top}(C \oplus$ $0) \mathbf{x}_{7}=0$ is satisfied in all eight cases, Corollary 4 shows that $B_{7}$ is invertible. Since $B_{6}=B_{1}+\mathbf{x}_{7} \mathbf{x}_{7}^{\top}+\mathbf{x}_{6} \mathbf{x}_{6}^{\top}$ and

$$
\begin{aligned}
\mathbf{x}_{6}^{\top}\left(B_{1}+\mathbf{x}_{7} \mathbf{x}_{7}^{\top}\right)^{-1} \mathbf{x}_{6} & =\mathbf{x}_{6}^{\top} B_{1}^{-1} \mathbf{x}_{6}+\left(\mathbf{x}_{6}^{\top} B_{1}^{-1} \mathbf{x}_{7}\right)\left(\mathbf{x}_{7}^{\top} B_{1}^{-1} \mathbf{x}_{6}\right) \\
& =\mathbf{x}_{6}^{\top}(C \oplus 0) \mathbf{x}_{6}+\mathbf{x}_{6}^{\top}(C \oplus 0) \mathbf{x}_{7}=0
\end{aligned}
$$

it follows that $B_{6}$ is invertible. Finally, $B_{5}=B_{1}+\mathbf{x}_{7} \mathbf{x}_{7}^{\top}+\mathbf{x}_{6} \mathbf{x}_{6}^{\top}+\mathbf{x}_{5} \mathbf{x}_{5}^{\top}$ and

$$
\begin{aligned}
\mathbf{x}_{5}^{\top}\left(B_{1}+\mathbf{x}_{7} \mathbf{x}_{7}^{\top}\right. & \left.+\mathbf{x}_{6} \mathbf{x}_{6}^{\top}\right)^{-1} \mathbf{x}_{5}=\mathbf{x}_{5}^{\top}\left(B_{1}+\mathbf{x}_{7} \mathbf{x}_{7}^{\top}\right)^{-1} \mathbf{x}_{5}+\left(\mathbf{x}_{5}^{\top}\left(B_{1}+\mathbf{x}_{7} \mathbf{x}_{7}^{\top}\right)^{-1} \mathbf{x}_{6}\right)^{2} \\
& =\mathbf{x}_{5}^{\top} B_{1}^{-1} \mathbf{x}_{5}+\left(\mathbf{x}_{5}^{\top} B_{1}^{-1} \mathbf{x}_{7}\right)^{2}+\left(\mathbf{x}_{5}^{\top} B_{1}^{-1} \mathbf{x}_{6}+\mathbf{x}_{5}^{\top} B_{1}^{-1} \mathbf{x}_{7} \mathbf{x}_{7}^{\top} B_{1}^{-1} \mathbf{x}_{6}\right)^{2} \\
& =\mathbf{x}_{5}^{\top} B_{1}^{-1}\left(\mathbf{x}_{5}+\mathbf{x}_{6}+\mathbf{x}_{7}\right)+\mathbf{x}_{5}^{\top} B_{1}^{-1} \mathbf{x}_{7} \mathbf{x}_{7}^{\top} B_{1}^{-1} \mathbf{x}_{6} \\
& =\mathbf{x}_{5}^{\top}(C \oplus 0)\left(\mathbf{x}_{5}+\mathbf{x}_{6}+\mathbf{x}_{7}\right)+\mathbf{x}_{5}^{\top}(C \oplus 0) \mathbf{x}_{7} \mathbf{x}_{7}^{\top}(C \oplus 0) \mathbf{x}_{6}=0
\end{aligned}
$$

show that $B_{5}$ is invertible. 


\section{Other properties and a problem on hamiltonicity}

A graph $\Gamma$ is vertex-transitive, edge-transitive, and arc-transitive, if its automorphism group acts transitively on the set of vertices, edges, and arcs of $\Gamma$, respectively. Recall that any arc-transitive graph is also vertex-transitive and edge-transitive. Let $d(u, v)$ denote the distance between two vertices $u, v$ of a connected graph $\Gamma$ and let $d:=$ $\max _{u, v \in V(\Gamma)} d(u, v)$ be its diameter. A connected graph $\Gamma$ is distance-regular if there exist numbers $p_{i j}^{k}(i, j, k \in\{0,1, \ldots, d\})$ such that for any pair of vertices $(u, v)$ with $d(u, v)=k$ the number of vertices $w \in V(\Gamma)$ with $d(u, w)=i$ and $d(w, v)=j$ equals $p_{i j}^{k}$. We refer to $[7,2]$ for more on vertex/edge/arc-transitive graphs and distance-regular graphs.

The number of matrices in $H G L_{n}\left(\mathbb{F}_{4}\right)$ is computed in [20, Corollary 5.32]. As observed in [6, Lemma 2.12], it can be also deduced from [2, Theorem 9.5.7]. In fact, graph $H_{n}\left(\mathbb{F}_{4}\right)$ is distance-regular with known parameters and distance $d(A, B)=\mathrm{rk}(A-B)$, so

$$
\left|H G L_{n}\left(\mathbb{F}_{4}\right)\right|=\prod_{j=0}^{n-1} \frac{2^{2 n}-2^{2 j}}{2^{j}\left(2^{j+1}+(-1)^{j}\right)}=2^{\frac{n(n-1)}{2}} \prod_{j=1}^{n}\left(2^{j}+(-1)^{j}\right) .
$$

The cardinality of $S G L_{n}\left(\mathbb{F}_{2}\right)$ is computed in [2, Lemma 9.5.9], which tells that

$$
\left|S G L_{n}\left(\mathbb{F}_{2}\right)\right|= \begin{cases}2^{\frac{n^{2}-1}{4}} \prod_{j=1}^{(n+1) / 2}\left(2^{2 j-1}-1\right) & \text { if } n \text { is odd } \\ 2^{\frac{(n+1)^{2}-1}{4}} \prod_{j=1}^{n / 2}\left(2^{2 j-1}-1\right) & \text { if } n \text { is even. }\end{cases}
$$

This number can be obtained also by combining Corollaries 3.31 and 4.10 in [20]. The graph, which has the vertex set formed by all (singular and invertible) alternate matrices and edges $\{A, B\}$ formed by matrices with $\operatorname{rk}(A-B)=2$, is distance-regular with known parameters. Moreover, the distance between any $A$ and $B$ is given by $d(A, B)=\operatorname{rk}(A-$ $B) / 2$. Hence, if $n$ is even, it is easy to deduce from [2, Theorem 9.5.6] that precisely

$$
\prod_{j=0}^{n / 2-1} \frac{2^{2 j}\left(2^{n-2 j-1}-1\right)\left(2^{n-2 j}-1\right)}{2^{2 j+2}-1}=2^{\frac{(n-1)^{2}-1}{4}} \prod_{j=1}^{n / 2}\left(2^{2 j-1}-1\right)=\frac{\left|S G L_{n}\left(\mathbb{F}_{2}\right)\right|}{2^{n}}
$$

matrices in $S G L_{n}\left(\mathbb{F}_{2}\right)$ are alternate. This is computed also in [20, Corollary 3.31].

Proposition 12. Let $n \geqslant 2$. Graph $H G L_{n}\left(\mathbb{F}_{4}\right)$ is arc-transitive of degree

$$
\frac{\left(2^{n}-(-1)^{n}\right)\left(2^{n-1}-(-1)^{n-1}\right)}{3} \text {. }
$$

If $n$ is odd, then $S G L_{n}\left(\mathbb{F}_{2}\right)$ is arc-transitive. If $n$ is even, then $S G L_{n}\left(\mathbb{F}_{2}\right)$ is not vertextransitive. It is not edge-transitive unless $n=2$. Nonalternate and alternate matrices have vertex degree $2^{n-1}-1$ and $2^{n}-1$, respectively.

To prove Proposition 12, we need an additional lemma.

Lemma 13. Let $n \geqslant 3$ be odd and assume that nonzero $\mathbf{x}, \mathbf{y} \in \mathbb{F}_{2}^{n}$ satisfy $\mathbf{x}^{\top} \mathbf{x}=0=\mathbf{y}^{\top} \mathbf{y}$. Then there is an invertible matrix $P$ such that $P^{\top}=P^{-1}$ and $P \mathbf{x}=\mathbf{y}$. 
Proof. It suffices to consider the case, where $\mathbf{x}=\mathbf{e}_{1}+\mathbf{e}_{2}$. In fact, then there are matrices $P_{1}$ and $P_{2}$ such that $P_{1}\left(\mathbf{e}_{1}+\mathbf{e}_{2}\right)=\mathbf{y}, P_{2}\left(\mathbf{e}_{1}+\mathbf{e}_{2}\right)=\mathbf{x}$ and $P_{j}^{\top}=P_{j}^{-1}$, so $P:=P_{1} P_{2}^{-1}$ solves the original problem.

Let $\mathbf{x}=\mathbf{e}_{1}+\mathbf{e}_{2}$ and $\mathbf{y}=:\left(y_{1}, \ldots, y_{n}\right)^{\top}$. Since $\mathbf{y}$ is nonzero, $\mathbf{y}^{\top} \mathbf{y}=0$, and $n$ is odd, there are $j_{1}, j_{2}$ such that $y_{j_{1}}=1$ and $y_{j_{2}}=0$. Let $\mathbf{z}:=\mathbf{e}_{j_{1}}$. Then $\mathbf{z}^{\top} \mathbf{z}=1=$ $\mathbf{z}^{\top} \mathbf{y}$, so vectors $\mathbf{x}_{1}:=\mathbf{y}-\mathbf{z}$ and $\mathbf{x}_{2}:=\mathbf{z}$ satisfy $\mathbf{x}_{1}^{\top} \mathbf{x}_{1}=1=\mathbf{x}_{2}^{\top} \mathbf{x}_{2}$ and $\mathbf{x}_{1}^{\top} \mathbf{x}_{2}=0$. Let $\rho: \mathbb{F}_{2}^{n} \rightarrow \mathbb{F}_{2}^{n}$ be the identity map, $f: \mathbb{F}_{2}^{n} \times \mathbb{F}_{2}^{n} \rightarrow \mathbb{F}_{2}^{n}$ a bilinear map defined by $f\left(\mathbf{w}_{1}, \mathbf{w}_{2}\right):=\mathbf{w}_{1}^{\top} \mathbf{w}_{2}, \mathbf{h}:=\sum_{j=1}^{n} \mathbf{e}_{j}$, and $\sigma: \operatorname{Lin}\left\{\mathbf{e}_{1}, \mathbf{e}_{2}\right\} \rightarrow \mathbb{F}_{2}^{n}$ an injective linear map defined by $\sigma\left(a_{1} \mathbf{e}_{1}+a_{2} \mathbf{e}_{2}\right):=a_{1} \mathbf{x}_{1}+a_{2} \mathbf{x}_{2}$. Then $f\left(\sigma\left(\mathbf{w}_{1}\right), \sigma\left(\mathbf{w}_{2}\right)\right)=f\left(\mathbf{w}_{1}, \mathbf{w}_{2}\right)$ for all $\mathbf{w}_{1}, \mathbf{w}_{2} \in \operatorname{Lin}\left\{\mathbf{e}_{1}, \mathbf{e}_{2}\right\}$. Since vector $\mathbf{h}=\rho(\mathbf{h})$ has nonzero third and $j_{2}$-th coordinate, we deduce that $\mathbf{h} \notin \operatorname{Lin}\left\{\mathbf{e}_{1}, \mathbf{e}_{2}\right\}$ and $\rho(\mathbf{h}) \notin \sigma\left(\operatorname{Lin}\left\{\mathbf{e}_{1}, \mathbf{e}_{2}\right\}\right)=\operatorname{Lin}\left\{\mathbf{x}_{1}, \mathbf{x}_{2}\right\}$. By a version of Witt's theorem for fields of characteristic two [17, Theorem 3], $\sigma$ can be extended to a bijective linear map $\sigma: \mathbb{F}_{2}^{n} \rightarrow \mathbb{F}_{2}^{n}$ such that $f\left(\sigma\left(\mathbf{w}_{1}\right), \sigma\left(\mathbf{w}_{2}\right)\right)=f\left(\mathbf{w}_{1}, \mathbf{w}_{2}\right)$ for all $\mathbf{w}_{1}, \mathbf{w}_{2} \in \mathbb{F}_{2}^{n}$. Let $\mathbf{x}_{j}:=\sigma\left(\mathbf{e}_{j}\right)$ for $j \geqslant 3$. Then $\mathbf{x}_{i}^{\top} \mathbf{x}_{i}=1$ and $\mathbf{x}_{i}^{\top} \mathbf{x}_{k}=0$ for all distinct $i, k \leqslant n$, so matrix $P$ with $\mathbf{x}_{j}$ as the $j$-th column satisfies $P^{\top} P=I$ and $P \mathbf{x}=\mathbf{y}$.

Proof of Proposition 12. We start with graph $S G L_{n}\left(\mathbb{F}_{2}\right)$. If $A \in S G L_{n}\left(\mathbb{F}_{2}\right)$ is alternate, then $\mathbf{x}^{\top} A^{-1} \mathbf{x}=0$ for all $\mathbf{x}$, so by Corollary 4 any nonzero $\mathbf{x}$ generate a neighbor $A+\mathbf{x} \mathbf{x}^{\top}$. Hence the degree of $A$ equals $2^{n}-1$. For nonalternate $A \in S G L_{n}\left(\mathbb{F}_{2}\right)$ we have computed the number of nonzero column vectors $\mathbf{x}$, that satisfy $\mathbf{x}^{\top} A^{-1} \mathbf{x}=0$, already in (11), so the degree of $A$ equals $\sum_{k=1}^{\lfloor n / 2\rfloor}\left(\begin{array}{c}n \\ 2 k\end{array}\right)=\sum_{j=0}^{n}\left(\begin{array}{c}n \\ j\end{array}\right)\left(\frac{1^{j} 1^{n-j}+(-1)^{j} 1^{n-j}}{2}\right)-1=2^{n-1}-1$. Therefore the graph $S G L_{n}\left(\mathbb{F}_{2}\right)$ is not regular for even $n$, since then both alternate and nonalternate matrices exist in $S G L_{n}\left(\mathbb{F}_{2}\right)$. Consequently it is neither vertex-transitive. The graph $S G L_{2}\left(\mathbb{F}_{2}\right)$ is a star on four vertices, so an edge-transitive graph. Let $n \geqslant 4$ be even, $A:=\sum_{j=1}^{n / 2}\left(E_{2 j-1,2 j}+E_{2 j, 2 j-1}\right), B_{1}:=A+E_{11}$, and $B_{2}:=A+E_{11}+E_{n n}$. Then $\left\{A, B_{1}\right\}$ and $\left\{B_{1}, B_{2}\right\}$ are both edges in $S G L_{n}\left(\mathbb{F}_{2}\right)$. Since $A$ is alternate, while $B_{1}$ and $B_{2}$ are not, no automorphism which maps $\left\{A, B_{1}\right\}$ to $\left\{B_{1}, B_{2}\right\}$ exists, i.e., the graph is not edgetransitive.

We next prove that graph $S G L_{n}\left(\mathbb{F}_{2}\right)$ for $n$ odd and graph $H G L_{n}\left(\mathbb{F}_{4}\right)$ are arc-transitive. Let $\left(A_{1}, B_{1}\right)$ and $\left(A_{2}, B_{2}\right)$ be two arcs. Then $B_{j}=A_{j}+\mathbf{x}_{j} \mathbf{x}_{j}^{\dagger}$ for some nonzero $\mathbf{x}_{j}$ with $\mathbf{x}_{j}^{\dagger} A_{j}^{-1} \mathbf{x}_{j}=0$. There is an invertible matrix $Q_{j}$ such that $A_{j}^{-1}=Q_{j} Q_{j}^{\dagger}$. Then $\mathbf{y}_{j}:=Q_{j}^{\dagger} \mathbf{x}_{j}$ satisfies $\mathbf{y}_{j}^{\dagger} \mathbf{y}_{j}=0$. By Lemma 13 in case of symmetric matrices, and by [13, Lemma 2.3] applied at $1 \times n$ matrices $\mathbf{y}_{1}^{*}, \mathbf{y}_{2}^{*}$ in hermitian case, there is an invertible matrix $P$ such that $P^{\dagger}=P^{-1}$ and $P \mathbf{y}_{1}=\mathbf{y}_{2}$. If $R:=\left(Q_{2}^{-1}\right)^{\dagger} P Q_{1}^{\dagger}$, then the automorphism $\Phi(X)=R X R^{\dagger}$ satisfies $\Phi\left(A_{1}\right)=A_{2}$ and $\Phi\left(B_{1}\right)=B_{2}$, so the graph is arc-transitive.

Finally we compute the degree in $H G L_{n}\left(\mathbb{F}_{4}\right)$. By vertex-transitivity, it suffices to count the neighbors of $I$. By Corollary 3 , any such matrix is of the form $I+\mathbf{x x}^{*}$, where $\mathbf{x}^{*} \mathbf{x}=0$ and $\mathbf{x} \neq 0$. Moreover, if $\mathbf{x}$ and $\mathbf{y}$ are linearly dependent and nonzero, then $\mathbf{x x}^{*}=\mathbf{y} \mathbf{y}^{*}$, so the degree of $I$ equals the cardinality of the hermitian variety $\left\{\operatorname{Lin}\{\mathbf{x}\}: \mathbf{x}^{*} \mathbf{x}=0, \mathbf{x} \neq 0\right\}$, which equals (23) by [3, Theorem 8.1].

It is well known that graphs $H G L_{2}\left(\mathbb{F}_{4}\right)$ and $S G L_{3}\left(\mathbb{F}_{2}\right)$, that is, the Petersen graph and the Coxeter graph are distance-regular. Graph $H G L_{3}\left(\mathbb{F}_{4}\right)$ is distance-regular as 
well [2, p. 364]. Moreover, these three graphs are all distance-transitive. It is obvious that $S G L_{n}\left(\mathbb{F}_{2}\right)$ is not distance-regular for $n$ even, since it is not regular. The counterpart for $n$ odd is shown below.

Proposition 14. Let $n \geqslant 4$. Then $H G L_{n}\left(\mathbb{F}_{4}\right)$ and $S G L_{n}\left(\mathbb{F}_{2}\right)$ are not distance-regular.

Proof. Pick $\mathbf{x}_{1}, \mathbf{y}_{1}, \mathbf{x}_{2}, \mathbf{y}_{2}$ such that $\mathbf{x}_{j}$ and $\mathbf{y}_{j}$ are linearly independent, $\mathbf{x}_{j}^{\dagger} \mathbf{x}_{j}=0=\mathbf{y}_{j}^{\dagger} \mathbf{y}_{j}$, $\mathbf{x}_{1}^{\dagger} \mathbf{y}_{1}=0$, and $\mathbf{x}_{2}^{\dagger} \mathbf{y}_{2}=1$. For example, we can take $\mathbf{x}_{1}=\mathbf{x}_{2}:=\mathbf{e}_{1}+\mathbf{e}_{2}, \mathbf{y}_{1}:=\mathbf{e}_{3}+\mathbf{e}_{4}$, and $\mathbf{y}_{2}:=\mathbf{e}_{2}+\mathbf{e}_{3}$. Then $I+\mathbf{x}_{j} \mathbf{x}_{j}^{\dagger}$ and $I+\mathbf{y}_{j} \mathbf{y}_{j}^{\dagger}$ are invertible by Corollary $3 /$ Corollary 4 , and are at distance two. We will show that $I$ is their unique common neighbor if $j=2$, while in case $j=1$ there is an additional common neighbor, given by the matrix $I+\mathbf{x}_{j} \mathbf{x}_{j}^{\dagger}+\mathbf{y}_{j} \mathbf{y}_{j}^{\dagger}$, so the graph is not distance-regular.

Let $A \neq I$ be a common neighbor of $I+\mathbf{x}_{j} \mathbf{x}_{j}^{\dagger}$ and $I+\mathbf{y}_{j} \mathbf{y}_{j}^{\dagger}$. Then $A=I+\mathbf{x}_{j} \mathbf{x}_{j}^{\dagger}+\mathbf{z}_{j} \mathbf{z}_{j}^{\dagger}=$ $I+\mathbf{y}_{j} \mathbf{y}_{j}^{\dagger}+\mathbf{w}_{j} \mathbf{w}_{j}^{\dagger}$ for some nonzero column vectors $\mathbf{z}_{j}, \mathbf{w}_{j}$ such that $\left\{\mathbf{x}_{j}, \mathbf{z}_{j}\right\}$ and $\left\{\mathbf{y}_{j}, \mathbf{w}_{j}\right\}$ are both pairs of linearly independent vectors. Consequently,

$$
\mathbf{x}_{j} \mathbf{x}_{j}^{\dagger}+\mathbf{y}_{j} \mathbf{y}_{j}^{\dagger}+\mathbf{z}_{j} \mathbf{z}_{j}^{\dagger}+\mathbf{w}_{j} \mathbf{w}_{j}^{\dagger}=0
$$

The linear span of $\mathbf{x}_{j}, \mathbf{y}_{j}, \mathbf{z}_{j}, \mathbf{w}_{j}$ is two-dimensional, since the opposite would mean that a sum of rank-three and rank-one matrix vanish in (24). Consequently, $\mathbf{z}_{j}, \mathbf{w}_{j} \in \operatorname{Lin}\left\{\mathbf{x}_{j}, \mathbf{y}_{j}\right\}$, so $\mathbf{z}_{j} \mathbf{z}_{j}^{\dagger}, \mathbf{w}_{j} \mathbf{w}_{j}^{\dagger}$ are among matrices $\mathbf{x}_{j} \mathbf{x}_{j}^{\top}, \mathbf{y}_{j} \mathbf{y}_{j}^{\top},\left(\mathbf{x}_{j}+\mathbf{y}_{j}\right)\left(\mathbf{x}_{j}+\mathbf{y}_{j}\right)^{\top}$ in symmetric case, and among $\mathbf{x}_{j} \mathbf{x}_{j}^{*}, \mathbf{y}_{j} \mathbf{y}_{j}^{*},\left(\mathbf{x}_{j}+\mathbf{y}_{j}\right)\left(\mathbf{x}_{j}+\mathbf{y}_{j}\right)^{*},\left(\mathbf{x}_{j}+\imath \mathbf{y}_{j}\right)\left(\mathbf{x}_{j}+\imath \mathbf{y}_{j}\right)^{*},\left(\mathbf{x}_{j}+\bar{\imath} \mathbf{y}_{j}\right)\left(\mathbf{x}_{j}+\bar{\imath} \mathbf{y}_{j}\right)^{*}$ in hermitian case. It is now easy to see that only $\mathbf{z}_{j} \mathbf{z}_{j}^{\dagger}=\mathbf{y}_{j} \mathbf{y}_{j}^{\dagger}$ and $\mathbf{w}_{j} \mathbf{w}_{j}^{\dagger}=\mathbf{x}_{j} \mathbf{x}_{j}^{\dagger}$ meet the criteria given by $(24)$ and $A \neq I$. Hence, $A=I+\mathbf{x}_{j} \mathbf{x}_{j}^{\dagger}+\mathbf{y}_{j} \mathbf{y}_{j}^{\dagger}$. Since $\mathbf{y}_{j}^{\dagger}\left(I+\mathbf{x}_{j} \mathbf{x}_{j}^{\dagger}\right)^{-1} \mathbf{y}_{j}=\mathbf{y}_{j}^{\dagger}\left(I+\mathbf{x}_{j} \mathbf{x}_{j}^{\dagger}\right) \mathbf{y}_{j}=$ $\mathbf{y}_{j}^{\dagger} \mathbf{x}_{j} \cdot \mathbf{x}_{j}^{\dagger} \mathbf{y}_{j}$, Corollary 3/Corollary 4 implies that $A$ is invertible if and only if $j=1$.

It follows from the proof of Proposition 14 that the girth of $H G L_{n}\left(\mathbb{F}_{4}\right)$ and $S G L_{n}\left(\mathbb{F}_{2}\right)$ equals 4 when $n \geqslant 4$. Recall from the proof of Theorems 7 and 8 that their odd girth, i.e. the length of the shortest odd cycle, equal 5 and 7 , respectively. It is well known that the girth of the Petersen and the Coxeter graph, that is the girth of $H G L_{2}\left(\mathbb{F}_{4}\right)$ and $S G L_{3}\left(\mathbb{F}_{2}\right)$, equal 5 and 7 , respectively. The graph $H G L_{3}\left(\mathbb{F}_{4}\right)$ has girth 5 . In fact, by $[2$, Theorem 11.3.7] its intersection array equals $\{9,8,6,3 ; 1,1,3,8\}$, so two vertices at distance two have just one common neighbor. Hence, there are no 4-cycles in $H G L_{3}\left(\mathbb{F}_{4}\right)$, while it contains 5-cycles, since it contains the Petersen graph $H G L_{2}\left(\mathbb{F}_{4}\right)$ as a subgraph.

When proving that a particular graph is a core, it is often useful if the automorphism group act transitively on pairs of vertices at distance two (cf. Lemma 1 and [8, Theorem 4.1]). This is not the case for graphs $H G L_{n}\left(\mathbb{F}_{4}\right)$ and $S G L_{n}\left(\mathbb{F}_{2}\right)$ if $n \geqslant 4$. In fact, it follows from the proof of Proposition 14 that the automorphism groups have at least two orbits if $n \geqslant 4$, since the vertices at distance two have either one or two common neighbors. For hermitian case we give a precise description of these orbits.

Proposition 15. Let $n \geqslant 4$. The automorphism group of $H G L_{n}\left(\mathbb{F}_{4}\right)$ has two orbits on 
2-arcs, which are given by

$$
\begin{array}{r}
\mathcal{O}_{1}=\left\{\left(A+\mathbf{x x}^{*}, A, A+\mathbf{y y}^{*}\right): A \in H G L_{n}\left(\mathbb{F}_{4}\right), \mathbf{x}, \mathbf{y} \in \mathbb{F}_{4}^{n}\right. \text { are linearly independent } \\
\left.\mathbf{x}^{*} A^{-1} \mathbf{x}=0=\mathbf{y}^{*} A^{-1} \mathbf{y}, \mathbf{x}^{*} A^{-1} \mathbf{y} \neq 0\right\}
\end{array}
$$

and

$$
\begin{array}{r}
\mathcal{O}_{2}=\left\{\left(A+\mathbf{x x}^{*}, A, A+\mathbf{y} \mathbf{y}^{*}\right): A \in H G L_{n}\left(\mathbb{F}_{4}\right), \mathbf{x}, \mathbf{y} \in \mathbb{F}_{4}^{n}\right. \text { are linearly independent } \\
\left.\mathbf{x}^{*} A^{-1} \mathbf{x}=0=\mathbf{y}^{*} A^{-1} \mathbf{y}, \mathbf{x}^{*} A^{-1} \mathbf{y}=0\right\}
\end{array}
$$

Proof. It follows from Corollary 3 that any 2-arc is of the form $\left(A+\mathbf{x x}^{*}, A, A+\mathbf{y y}^{*}\right)$ for some $A \in H G L_{n}\left(\mathbb{F}_{4}\right)$ and nonzero $\mathbf{x}, \mathbf{y}$ such that $\mathbf{x}^{*} A^{-1} \mathbf{x}=0=\mathbf{y}^{*} A^{-1} \mathbf{y}$. Moreover, $\mathbf{x}$ and $\mathbf{y}$ are linearly independent, since $A+\mathbf{x x}^{*} \neq A+\mathbf{y y}^{*}$.

Assume firstly that $l_{j}:=\left(A_{j}+\mathbf{x}_{j} \mathbf{x}_{j}^{*}, A_{j}, A_{j}+\mathbf{y}_{j} \mathbf{y}_{j}^{*}\right) \in \mathcal{O}_{1}$ for $j=1,2$. Pick an invertible matrix $Q_{j}$ such that $A_{j}^{-1}=Q_{j} Q_{j}^{*}$. Then $\mathbf{z}_{j}:=Q_{j}^{*} \mathbf{x}_{j}$ and $\mathbf{w}_{j}:=Q_{j}^{*} \mathbf{y}_{j}$ satisfy $\mathbf{z}_{j}^{*} \mathbf{z}_{j}=0=\mathbf{w}_{j}^{*} \mathbf{w}_{j}$ and $\mathbf{z}_{j}^{*} \mathbf{w}_{j} \neq 0$. By [13, Lemma 3.10] there exist an invertible matrix $P$ and nonzero $b \in \mathbb{F}_{4}$ such that $P^{*}=P^{-1}, P \mathbf{z}_{1}=\mathbf{z}_{2}$, and $P \mathbf{w}_{1}=b \mathbf{w}_{2}$. Since $b \bar{b}=1$, the matrix $R:=\left(Q_{2}^{-1}\right)^{*} P Q_{1}^{*}$ induce an automorphism $\Phi(X):=R X R^{*}$, which maps $l_{1}$ to $l_{2}$.

If $l_{1}, l_{2} \in \mathcal{O}_{2}$, then we produce an automorphism $\Phi$ that satisfies $\Phi\left(l_{1}\right)=l_{2}$ in the same way as above. We just replace [13, Lemma 3.10] by [13, Lemma 3.9].

If $n \in\{2,3\}$, then $\mathcal{O}_{2}=\emptyset$ by [3, Theorem 9.1]. Proposition 15 shortens the proof of Theorem 7 , since to apply Lemma 1 we only need to check that the two 2 -arcs

$$
\begin{aligned}
\left(I+\left(\mathbf{e}_{1}+\imath \mathbf{e}_{2}\right)\left(\mathbf{e}_{1}+\imath \mathbf{e}_{2}\right)^{*}, I, I+\left(\mathbf{e}_{1}+\mathbf{e}_{2}\right)\left(\mathbf{e}_{1}+\mathbf{e}_{2}\right)^{*}\right) & \in \mathcal{O}_{1}, \\
\left(I+\left(\mathbf{e}_{3}+\mathbf{e}_{4}\right)\left(\mathbf{e}_{3}+\mathbf{e}_{4}\right)^{*}, I, I+\left(\mathbf{e}_{1}+\mathbf{e}_{2}\right)\left(\mathbf{e}_{1}+\mathbf{e}_{2}\right)^{*}\right) & \in \mathcal{O}_{2}
\end{aligned}
$$

lie in 5-cycles. These are generated by matrices

$$
\left[\begin{array}{ll}
0 & \bar{\imath} \\
\imath & 0
\end{array}\right] \oplus I_{n-2},\left[\begin{array}{ll}
1 & 0 \\
0 & 1
\end{array}\right] \oplus I_{n-2},\left[\begin{array}{ll}
0 & 1 \\
1 & 0
\end{array}\right] \oplus I_{n-2},\left[\begin{array}{ll}
1 & 1 \\
1 & 0
\end{array}\right] \oplus I_{n-2},\left[\begin{array}{ll}
0 & \bar{z} \\
2 & 1
\end{array}\right] \oplus I_{n-2}
$$

and

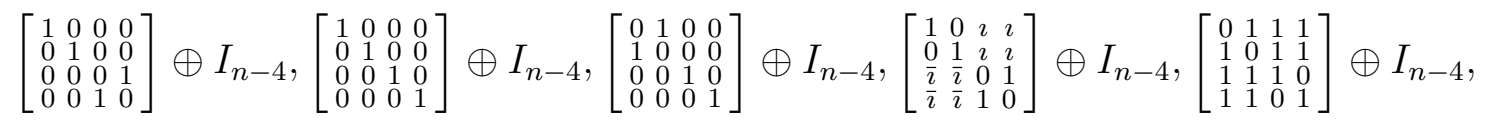

respectively.

There are only five known connected vertex-transitive graphs without a Hamilton cycle: the complete graph on two vertices, the Petersen graph, the Coxeter graph, and two graphs derived from the Petersen and Coxeter graphs by replacing each vertex with a triangle (see the survey paper [9] and the references therein). It is believed by many mathematicians that no other exist. Since the graphs $H G L_{n}\left(\mathbb{F}_{4}\right)$ and $S G L_{2 n-1}\left(\mathbb{F}_{2}\right)$ are vertex-transitive by Proposition 12 and they generalize the Petersen and the Coxeter graph in a natural way, it seems worthwhile to check if these graphs have a Hamilton cycle. 
Unfortunately we did not succeed in this task. With an application of the Concorde TSP Solver [5] we were able to find a Hamilton cycle in $H G L_{3}\left(\mathbb{F}_{4}\right), S G L_{4}\left(\mathbb{F}_{2}\right)$, and $S G L_{5}\left(\mathbb{F}_{2}\right)$, which are graphs on 280,448, and 13888 vertices, respectively. We state the general case, which include non-vertex-transitive graphs $S G L_{2 n}\left(\mathbb{F}_{2}\right)$, as an open problem.

Open Problem 16. Do graphs $H G L_{n}\left(\mathbb{F}_{4}\right)$ and $S G L_{n}\left(\mathbb{F}_{2}\right)$ contain a Hamilton cycle? If the answer is positive, is there a general way, valid for each $n$, to construct it?

The corresponding problems for graphs $H_{n}\left(\mathbb{F}_{4}\right)$ and $S_{n}\left(\mathbb{F}_{2}\right)$, which are formed by all singular and invertible matrices, is much easier. In fact, these graphs are Cayley graphs over abelian groups $\left(H_{n}\left(\mathbb{F}_{4}\right),+\right)$ and $\left(S_{n}\left(\mathbb{F}_{2}\right),+\right)$, respectively, where the generating set is formed by all matrices of rank one. Hence, these graphs contain a Hamilton cycle by [11, Corollary 3.2] or by [10, Exercise 12.17].

Unlike the graph $S G L_{2 n}\left(\mathbb{F}_{2}\right)$, its subgraph that is induced by nonalternating matrices is vertex-transitive. So studying the hamiltonicity of these subgraphs seems meaningful as well. We mention here just that in the case of $4 \times 4$ matrices we obtain a graph on 420 vertices, which has a Hamilton cycle.

Since the problem of whether connected Cayley graphs (other than the complete graph on two vertices) are Hamiltonian is also well studied (cf. [9]), another interesting question is whether $H G L_{n}\left(\mathbb{F}_{4}\right)$ and $S G L_{2 n-1}\left(\mathbb{F}_{2}\right)$ are Cayley graphs. Though we believe that this is not the case, the problem seems nontrivial. Since a vertex-transitive graph is a Cayley graph if and only if its automorphism group contains a regular subgroup [18], a related problem is to find a characterization of all automorphisms $=$ endomorphisms (= adjacency preservers in the language of matrix theory) of graphs $H G L_{n}\left(\mathbb{F}_{4}\right)$ and $S G L_{n}\left(\mathbb{F}_{2}\right)$. More precisely, the question is whether an analogous result to [14] holds, and the only automorphisms of $S G L_{n}\left(\mathbb{F}_{2}\right)$ are those of the form

$$
A \mapsto P A P^{\top} \quad \text { or } \quad A \mapsto P A^{-1} P^{\top},
$$

and the only automorphisms of $H G L_{n}\left(\mathbb{F}_{4}\right)$ are those of the form

$$
A \mapsto P A^{\varphi} P^{*} \quad \text { or } \quad A \mapsto P\left(A^{\varphi}\right)^{-1} P^{*},
$$

where $P$ is an invertible matrix and $\varphi \in\{-$,identity map $\}$ is applied entry-wise. For the Coxeter graph $S G L_{3}\left(\mathbb{F}_{2}\right)$ and the Petersen graph $H G L_{2}\left(\mathbb{F}_{4}\right)$ this is indeed the case. In fact, it is not difficult to see that all maps in (25) are distinct if $n \geqslant 3$. Since there are precisely 168 invertible matrices $P$ over $\mathbb{F}_{2}$ of size $3 \times 3$ (cf. [20, Theorem 1.6]), there are in total $2 \cdot 168=336$ maps of the form (25), which is the same as the order of the well known automorphism group of the Coxeter graph. In the case of $H G L_{2}\left(\mathbb{F}_{4}\right)$ it is easy to see that $A^{-1}=Q \bar{A} Q^{*}$ for $Q=\left[\begin{array}{ll}0 & 1 \\ 1 & 0\end{array}\right]$, and two maps $A \mapsto P_{1} A^{\varphi_{1}} P_{1}^{*}, A \mapsto P_{2} A^{\varphi_{2}} P_{2}^{*}$ are the same if and only if $\varphi_{1}=\varphi_{2}$ and $P_{1} \in\left\{P_{2}, \imath P_{2}, \bar{\imath} P_{2}\right\}$. Since there are 180 invertible matrices $P$ over $\mathbb{F}_{4}$ of size $2 \times 2$ (cf. [20, Theorem 1.6]), there are in total $\frac{2 \cdot 180}{3}=120$ maps of the form (26), which is the same as the order of the well known automorphism group of the Petersen graph. To see that all the maps in (25) and (26) are automorphisms indeed, note that $A^{-1}-B^{-1}=A^{-1}(B-A) B^{-1}$ for invertible matrices, so $\operatorname{rk}\left(A^{-1}-B^{-1}\right)=\operatorname{rk}(A-B)$. Remark 17. Open Problem 16 was firstly posed by the present author at Joint Mathematical Conference CSASC in June 2013, in Koper, Slovenia. 


\section{Acknowledgements}

The author would like to thank the reviewer for his comments that improved the presentation of the paper. The author was supported in part by a postdoctoral grant at University of Primorska and by grant P1-0222 of the Slovenian Research Agency.

\section{References}

[1] A. Blunck, P. Lévay, M. Saniga, and P. Vrana. Invertible symmetric $3 \times 3$ binary matrices and GQ(2,4). Linear Multilinear Algebra 60(10):1143-1154, 2012.

[2] A. E. Brouwer, A. M. Cohen, and A. Neumaier. Distance-regular graphs. Ergebnisse der Mathematik und ihrer Grenzgebiete (3), 18, Springer-Verlag, Berlin, 1989.

[3] R. C. Bose and I. M. Chakravarti. Hermitian varieties in a finite projective space $\operatorname{PG}\left(N, q^{2}\right)$. Canad. J. Math. 18:1161-1182, 1966.

[4] P. J. Cameron and P. A. Kazanidis. Cores of symmetric graphs. J. Aust. Math. Soc. 85(2):145-154, 2008.

[5] Concorde TSP Solver. Available at http://www . math. uwaterloo.ca/tsp/concorde.html. Accessed 26 September 2013.

[6] G. Dolinar, A. Guterman, B. Kuzma, and M. Orel. Permanent versus determinant over a finite field. Sovrem. Mat. Prilozh. Vol. 80, Proceedings of the International Conference "Modern Algebra and Its Applications" (Batumi, 2011), Part 1, 2012. English translation: J. Math. Sci. (N. Y.) 193(3):404-413, 2013.

[7] C. Godsil and G. F. Royle. Algebraic graph theory. Graduate Texts in Mathematics, 207. Springer-Verlag, New York, 2001.

[8] C. Godsil and G. F. Royle. Cores of geometric graphs. Ann. Comb. 15(2):267-276, 2011.

[9] K. Kutnar and D. Marušič. Hamilton cycles and paths in vertex-transitive graphscurrent directions. Discrete Math. 309(17):5491-5500, 2009.

[10] L. Lovász. Combinatorial problems and exercises. Second edition. North-Holland Publishing Co., Amsterdam, 1993.

[11] D. Marušič. Hamiltonian circuits in Cayley graphs. Discrete Math. 46(1):49-54, 1983.

[12] M. Orel. A note on adjacency preservers on Hermitian matrices over finite fields. Finite Fields Appl. 15(4):441-449, 2009.

[13] M. Orel. Adjacency preservers on invertible hermitian matrices I. Linear Algebra Appl. (2014), doi:10.1016/j.laa.2014.10.034

[14] M. Orel. Adjacency preservers on invertible hermitian matrices II. Linear Algebra Appl. (2014), doi:10.1016/j.laa.2014.10.033

[15] M. Orel. Adjacency preservers, symmetric matrices, and cores. J. Algebraic Combin. 35(4):633-647, 2012. 
[16] M. Orel. Ohranjevalci na matričnih prostorih. PhD Thesis (in Slovenian), Ljubljana, 2009.

[17] V. Pless. On Witt's theorem for nonalternating symmetric bilinear forms over a field of characteristic 2. Proc. Amer. Math. Soc. 15:979-983, 1964.

[18] G. Sabidussi. On a class of fixed-point-free graphs. Proc. Amer. Math. Soc. 9:800-804, 1958.

[19] M. Tsatsomeros. Matrix Equalities and Inequlaities. In: L. Hogben (Ed.), R. Brualdi, A. Greenbaum, and R. Mathias (Associate Eds.). Handbook of Linear Algebra. Discrete Mathematics and its Applications (Boca Raton). Chapman \& Hall/CRC, Boca Raton, FL, 2007.

[20] Z.-X. Wan. Geometry of classical groups over finite fields. Studentlitteratur, Lund; Chartwell-Bratt Ltd., Bromley, 1993.

[21] Z.-X. Wan. Geometry of matrices. In memory of Professor L. K. Hua (1910-1985). World Scientific Publishing Co., Inc., River Edge, NJ, 1996.

[22] Z.-X. Wan. The graph of binary symmetric matrices of order 3. Northeast. Math. J. 11(1):1-2, 1995. 


\title{
Corrigendum: On generalizations of the Petersen graph and the Coxeter graph
}

\author{
Marko Orel \\ University of Primorska, FAMNIT, Glagoljaška 8, 6000 Koper, Slovenia \\ IMFM, Jadranska 19, 1000 Ljubljana, Slovenia \\ University of Primorska, IAM, Muzejski trg 2, 6000 Koper, Slovenia \\ marko.orel@upr.si
}

Submitted: Sep 27, 2013; Accepted: Nov 4, 2015; Published: Nov 13, 2015

Mathematics Subject Classifications 2010: 05C50, 15B33, 15B57

\section{Corrigendum}

The figures in the original paper (Figure 1 and Figure 2) were missing detail at their vertices. In particular, there were supposed to be matrices overlaid at each vertex which we give below.

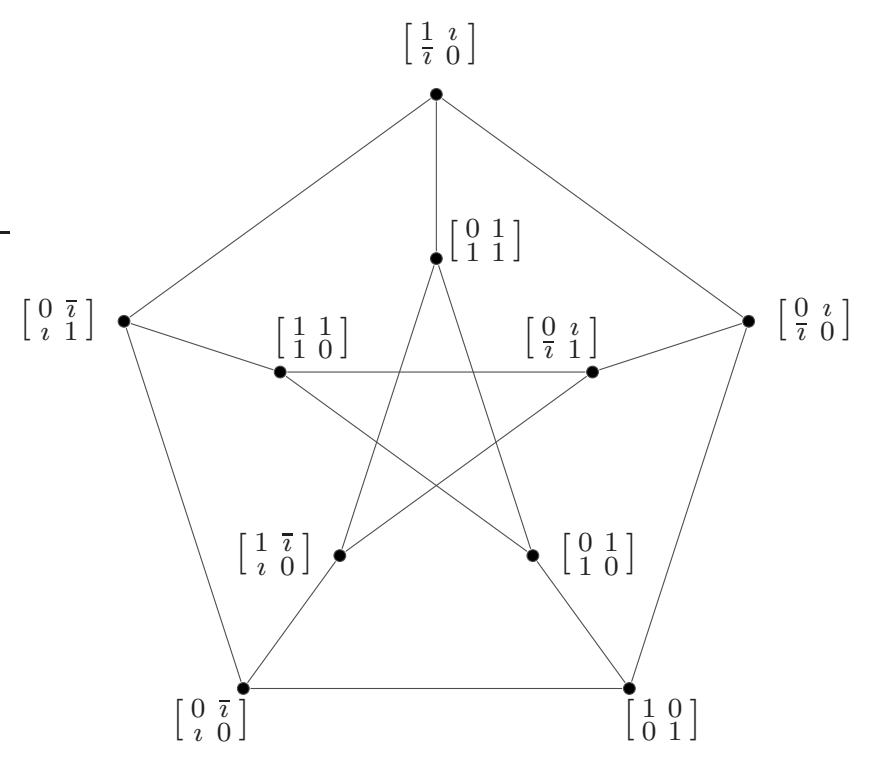

Figure 1: Graph $\mathrm{HGL}_{2}\left(\mathbb{F}_{4}\right)$ is the Petersen graph. 


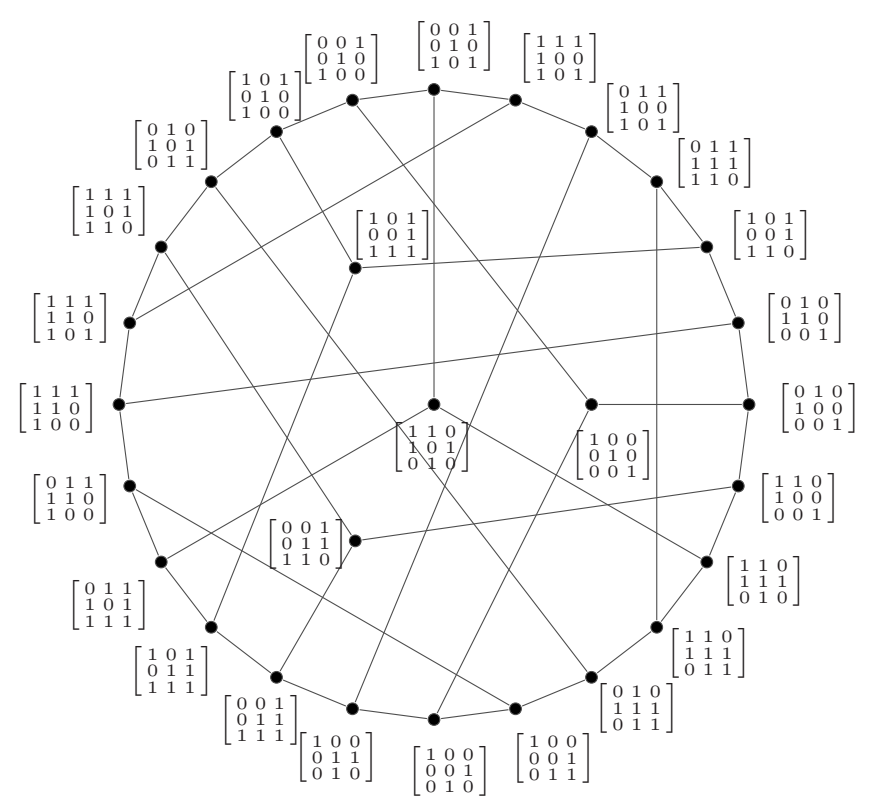

Figure 2: Graph $S G L_{3}\left(\mathbb{F}_{2}\right)$ is the Coxeter graph. 